\title{
Correction to "The 'Measuring Outcomes of Clinical Connectivity' (MOCC) Trial: Investigating Data Entry Errors in the Electronic Primary Care Research Network (ePCRN)"
}

In the abovementioned article (Fontaine P, Mendenhall TJ, Peterson K, Speedie SM.J Am Board Fam Med 2007;20(2):151-159), a name was misspelled in the acknowledgments: Theo Arventis, PhD, should be Theo Arvanitis, PhD. (doi: 10.3122/jabfm.2007.04.070112.)

\section{Correction to "Obesity Evaluation and Intervention During Family Medicine Well Visits"}

In the abovementioned article (Boardley D, Sherman C, Ambrosetti L, Lewis J.J Am Board Fam Med 2007;20(3):252-257), the author's credentials appeared incorrectly: Christopher Sherman, MS should have read Christopher Sherman, MD. We apologize for this error, and we regret any confusion or inconvenience it may have caused. (doi: 10.3122/jabfm.2007.04.070138.) 